\title{
Permanents of Hessenberg $(0,1)$-matrices
}

\author{
D.D. Olesky \\ Department of Computer Science \\ University of Victoria \\ Victoria, BC V8W 3P6 Canada \\ dolesky@cs.uvic.ca \\ Bryan Shader \\ Department of Mathematics \\ University of Wyoming \\ Laramie, WY 82071 \\ bshader@uwyo.edu \\ P. van den Driessche \\ Department of Mathematics \\ University of Victoria \\ Victoria, BC V8W 3P4 Canada \\ pvdd@math.uvic.ca
}

Submitted: Apr 22, 2005; Accepted: Dec 6, 2005; Published: Dec 13, 2005

Mathematics Subject Classifications: 05C50

\begin{abstract}
Let $P(m, n)$ denote the maximum permanent of an $n$-by-n lower Hessenberg $(0,1)$-matrix with $m$ entries equal to 1 . A "staircased" structure for some matrices achieving this maximum is obtained, and recursive formulas for computing $P(m, n)$ are given. This structure and results about permanents are used to determine the exact values of $P(m, n)$ for $n \leq m \leq 8 n / 3$ and for all $\operatorname{nnz}\left(H_{n}\right)-\operatorname{nnz}\left(H_{\lfloor n / 2\rfloor}\right) \leq$ $m \leq \operatorname{nnz}\left(H_{n}\right)$, where $\operatorname{nnz}\left(H_{n}\right)=\left(n^{2}+3 n-2\right) / 2$ is the maximum number of ones in an $n$-by- $n$ Hessenberg $(0,1)$-matrix.
\end{abstract}

\section{Introduction}

A transversal of an $n$-by- $n(0,1)$-matrix $A=\left[a_{i j}\right]$ is a collection of $n$ entries of $A$ equal to 1 , no two of which are in the same row or column. The permanent of $A$, denoted per $A$, is the number of distinct transversals of $A$. Equivalently,

$$
\text { per } A=\sum_{\sigma} a_{1 \sigma(1)} a_{2 \sigma(2)} \cdots a_{n \sigma(n)} \text {, }
$$


where the sum is over all permutations $\sigma$ of $\{1,2, \ldots, n\}$. We refer the reader to $[\mathrm{M}]$ for classic results, and to $[\mathrm{CW}]$ for a survey of recent research on permanents. A matrix $A$ is a lower Hessenberg matrix if $a_{i j}=0$ whenever $j \geq i+2$. Throughout the remainder of the paper, we abbreviate lower Hessenberg to Hessenberg. Let $\mathcal{H}(m, n)$ denote the set of $n$-by- $n$ Hessenberg $(0,1)$-matrices with $m$ entries equal to 1 , and let $P(m, n)$ denote the maximum permanent of a matrix in $\mathcal{H}(m, n)$.

In [BR, Ch. 7], computation of the permanent of an arbitrary rectangular matrix is considered. Additionally, upper and lower bounds for the permanent of such a $(0,1)$ matrix $A$ are given in terms of the number of ones in each row of $A$, the number of ones in each column of $A$, or the total number of ones in $A$. In [SHRC, Th. 2.3, 2.5], the maximum value of the permanent of a $p$-by- $q(0,1)$-matrix with $m$ entries equal to 1 for $p q-\max \{p, q\} \leq m \leq p q-2$ is given, and matrices attaining this value are determined. In [BGM, Th. 2.2], the maximum value of the permanent of an $n$-by- $n(0,1)$-matrix with $m$ entries equal to 1 for $n \leq m \leq 2 n$ is determined, and we observe that every matrix achieving this maximum is combinatorially equivalent to a Hessenberg matrix. In addition, the matrices attaining the maximum value of the permanent of an $n$-by- $n$ $(0,1)$-matrix for $n^{2}-2 n \leq m \leq n^{2}$ are determined. In this paper, we focus on Hessenberg matrices and determine the exact value of $P(m, n)$ for $n \geq 2$ and various values of $m$ with $n \leq m \leq \frac{n^{2}+3 n-2}{2}$.

We first state some notation and terminology (see $[\mathrm{BR}]$ for further details). The number of nonzero entries of the matrix $A$ is denoted by $\operatorname{nnz}(A)$. For integers $i$ and $j$ with $i \leq j$, denote $\{i, i+1, \ldots, j\}$ by $\langle i, j\rangle$, with $\{i\}=\langle i\rangle$ abbreviated to $i$. The submatrix of $A$ with entries from rows $\left\langle i_{1}, i_{2}\right\rangle$ and columns $\left\langle j_{1}, j_{2}\right\rangle$ is denoted by $A\left[\left\langle i_{1}, i_{2}\right\rangle,\left\langle j_{1}, j_{2}\right\rangle\right]$, with $A\left[\left\langle i_{1}, i_{2}\right\rangle,\left\langle i_{1}, i_{2}\right\rangle\right]$ abbreviated to $A\left[\left\langle i_{1}, i_{2}\right\rangle\right]$. Similarly, the submatrix of $A$ obtained by deleting rows $\left\langle i_{1}, i_{2}\right\rangle$ and columns $\left\langle j_{1}, j_{2}\right\rangle$ is denoted by $A\left(\left\langle i_{1}, i_{2}\right\rangle,\left\langle j_{1}, j_{2}\right\rangle\right)$, with $A\left(\left\langle i_{1}, i_{2}\right\rangle,\left\langle i_{1}, i_{2}\right\rangle\right)$ abbreviated to $A\left(\left\langle i_{1}, i_{2}\right\rangle\right)$.

The matrix $A$ is partly decomposable if there exist permutation matrices $P$ and $Q$ such that $P A Q$ has the form

$$
\left[\begin{array}{ll}
B & O \\
C & D
\end{array}\right],
$$

where $B$ and $D$ are square (nonvacuous) matrices. Equivalently, $A$ is partly decomposable if and only if it contains a zero submatrix with dimensions summing to $n$. If $A$ is not partly decomposable, then $A$ is fully indecomposable. If per $A>0$, then there exist permutation matrices $P$ and $Q$, and an integer $b$ such that $P A Q$ has the form

$$
\left[\begin{array}{ccccc}
A_{1} & O & O & \cdots & O \\
A_{21} & A_{2} & O & \cdots & O \\
\vdots & & \ddots & & \vdots \\
A_{b-1,1} & A_{b-2,2} & & A_{b-1} & O \\
A_{b 1} & A_{b 2} & \cdots & A_{b, b-1} & A_{b}
\end{array}\right]
$$

where the matrices $A_{1}, \ldots, A_{b}$ are fully indecomposable. The $n_{i}$-by- $n_{i}$ matrices $A_{i}$ are the fully indecomposable components of $A$ and are unique up to permutation of rows 
and columns. Note that per $A=\prod_{i=1}^{b}$ per $A_{i}$. The matrix $A$ has total support provided per $A(i, j)>0$ for all $i$ and $j$ such that $a_{i j}=1$; i.e., every nonzero entry of $A$ is on some transversal.

\section{Preliminary Results}

In this section we develop some basic preliminary results concerning the structure and permanents of matrices in $\mathcal{H}(m, n)$. The following shows that the fully indecomposable components of a Hessenberg matrix are each permutationally equivalent to a Hessenberg matrix.

Lemma 2.1 Let $A=\left[a_{i j}\right]$ be an n-by-n Hessenberg $(0,1)$-matrix with per $A>0$. Then each fully indecomposable component of $A$ is permutationally equivalent to a Hessenberg matrix.

Proof. The proof is by induction on $n$, with the result clearly true for $n=1$. Without loss of generality, assume that $A$ has total support. Since per $A>0$, column $n$ of $A$ contains at least one 1 . Let $B$ be the fully indecomposable component of $A$ that intersects column $n$.

If there is some $j$ such that $a_{j, j+1}=0$, then the fully indecomposable components of $A$ are those of $A[\langle 1, j\rangle]$ and $A[\langle j+1, n\rangle]$, and applying induction to each of these matrices yields that each fully indecomposable component of $A$ is permutationally equivalent to a Hessenberg matrix. If $a_{n n}=0$, then the fully indecomposable components of $A$ are the 1-by-1 matrix $\left[a_{n-1, n}\right]$, and those of the Hessenberg matrix $A(n-1, n)$. Again the inductive hypothesis applies, and hence each fully indecomposable component of $A$ is permutationally equivalent to a Hessenberg matrix. A similar argument handles the case that $a_{11}=0$.

Now assume that $a_{11}=1, a_{n n}=1$ and $a_{j, j+1}=1$ for $j=1,2, \ldots, n-1$. If each column of $A$ is a column of $B$, then $B=A$, and clearly the fully indecomposable component (namely $B$ ) of $A$ is Hessenberg. Otherwise, some column of $A$ does not intersect the columns of $B$. Let $j$ be the largest integer such that column $j$ of $A$ does not intersect the columns of $B$. Note $j<n$. Since $B$ intersects columns $j+1, \ldots, n$ of $A, B$ must contain each of the entries in positions $(j, j+1),(j+1, j+2), \ldots,(n-1, n)$ and $(n, n)$ of $A$ (otherwise $B$ would be partly decomposable). This implies that $B$ intersects rows $j, \ldots, n$. If there is some $i \geq j$ such that $a_{i j}=1$, then the fully indecomposable component that contains $a_{i j}$ has a row in common with $B$, and hence must be equal to $B$. But $B$ does not intersect column $j$. So $a_{i j}=0$ for $i=j, j+1, \ldots, n$. Now column $j$ has just one 1 , namely $a_{j-1, j}=1$. Hence the 1-by-1 matrix $\left[a_{j-1, j}\right]$ is a fully indecomposable component of $A$. It follows that the fully indecomposable components of $A$ are $\left[a_{j-1, j}\right]$ and the fully indecomposable components of $A(j-1, j)$. As $A(j-1, j)$ is Hessenberg, the inductive hypothesis applies. Hence each fully indecomposable component of $A$ is permutationally equivalent to a Hessenberg matrix. 
A Hessenberg $(0,1)$-matrix $A$ is staircased if whenever $i \geq j$ and $a_{i j}=0$, then $a_{k j}=0$ for $k=i+1, \ldots, n$ and $a_{i l}=0$ for $l=1, \ldots, j-1$. Note that if $A$ is staircased and $a_{i j}=0$, then $a_{k l}=0$ for all $i \leq k \leq n$ and $1 \leq l \leq j$.

Lemma 2.2 The following hold for an n-by-n Hessenberg $(0,1)$-matrix $A=\left[a_{i j}\right]$ :

(a) If $A$ is fully indecomposable, then $a_{11}=1, a_{n n}=1$ and $a_{i, i+1}=1$ for $i=1,2, \ldots, n-$ 1 .

(b) If $A$ is fully indecomposable and staircased, then $a_{i+1, i}=1$ for $i=1,2, \ldots, n-1$, and $a_{i i}=1$ for $i=1,2, \ldots, n$.

(c) If each $a_{i, i+1}=1(i=1,2, \ldots, n-1)$ and $k$ and $l$ are integers such that $n \geq k \geq$ $l \geq 1$, then

$$
\text { per } A(k, l)=\text { per } A[\langle 1, l-1\rangle] \text { per } A[\langle k+1, n\rangle] \text {, }
$$

in which a vacuous permanent with $l=1$ or $k=n$ is set equal to 1 .

Proof. If there is a $j$ with $a_{j, j+1}=0$, then $A[\langle 1, j\rangle,\langle j+1, n\rangle]$ is a zero submatrix of $A$ with dimensions summing to $n$, and hence $A$ is not fully indecomposable. If $a_{11}=0$ or $a_{n n}=0$, then $A$ has a row or column with a single 1 , and hence $A$ is not fully indecomposable. These observations prove (a).

If there is an $i$ such that $a_{i+1, i}=0$, then (since $A$ is staircased) $A[\langle i+1, n\rangle,\langle 1, i\rangle]=O$, and hence $A$ is not fully indecomposable. Similarly, if there is an $i$ such that $a_{i i}=0$, then (since $A$ is staircased) $A[\langle i, n\rangle,\langle 1, i\rangle]=O$, and hence $A$ is not fully indecomposable. This proves (b).

Statement (c) follows by noting that $A(k, l)$ has the form

$$
\left[\begin{array}{ccc}
A[\langle 1, l-1\rangle] & O & O \\
* & A[\langle l, k-1\rangle,\langle l+1, k\rangle] & O \\
* & * & A[\langle k+1, n\rangle]
\end{array}\right]
$$

and that $A[\langle l, k-1\rangle,\langle l+1, k\rangle]$ is a lower triangular (possibly vacuous) matrix with each of its main diagonal entries equal to 1 .

We now show that $\mathcal{H}(m, n)$ contains a special type of matrix with maximum permanent. For a Hessenberg $(0,1)$-matrix $A$, an interchangeable column pair of $A$ is a pair of entries $(k, l)$ and $(k-1, l)$ with $k>l$ such that $a_{k l}=1$ and $a_{k-1, l}=0$. An interchangeable row pair of $A$ is a pair of entries $(k, l)$ and $(k, l+1)$ with $k>l$ such that $a_{k l}=1$ and $a_{k, l+1}=0$.

Theorem 2.3 Let $m$ and $n$ be positive integers with $n \leq m \leq \frac{n^{2}+3 n-2}{2}$. Then there exists a matrix $A \in \mathcal{H}(m, n)$ with permanent $P(m, n)$ such that $A$ has the form $(1)$, where each $A_{i}$ is a fully indecomposable staircased Hessenberg matrix. 
Proof. Let $A \in \mathcal{H}(m, n)$ with per $A=P(m, n)$. By Lemma 2.1, assume that $A$ has the form (1), where each $A_{i}$ is a fully indecomposable Hessenberg matrix. We prove by induction on $n$ that there is a matrix in $\mathcal{H}(m, n)$ with permanent $P(m, n)$ having each fully indecomposable component staircased. This is clearly true for $n=1$.

First suppose that $b \geq 2$. Since per $A=\prod_{i=1}^{b}$ per $A_{i}$, it follows that per $A_{i}=P\left(\operatorname{nnz}\left(A_{i}\right), n_{i}\right)$ for $i=1,2, \ldots, b$. By induction, each $A_{i}$ is staircased.

Next suppose that $b=1$, that is, $A$ is fully indecomposable. We construct a sequence of matrices $B_{r} \in \mathcal{H}(m, n)$ as follows:

(a) $B_{0} \longleftarrow A$

(b) $r \longleftarrow 0$

(c) While ( $B_{r}$ is fully indecomposable and has an interchangeable row or column pair) do:

(c1) If $B_{r}$ has an interchangeable column pair, then choose such a pair $(k, l)$, $(k-1, l)$ with $l$ largest, and define $B_{r+1}$ to be the matrix obtained from $B_{r}$ by interchanging the 1 in position $(k, l)$ with the 0 in position $(k-1, l)$.

(c2) Else if $B_{r}$ has an interchangeable row pair, then choose such a pair $(k, l)$, $(k, l+1)$ with $k$ smallest, and define $B_{r+1}$ to be the matrix obtained from $B_{r}$ by interchanging the 1 in position $(k, l)$ with the 0 in position $(k, l+1)$.

(c3) $r \longleftarrow r+1$.

Note that this algorithm terminates since $B_{r+1}$ is either partly decomposable (in which case the algorithm is applied to the smaller fully indecomposable components) or remains fully indecomposable with fewer pairs $(i, j)$ and $\left(i^{\prime}, j^{\prime}\right)$ than $B_{r}$ such that $j^{\prime} \leq j \leq i \leq i^{\prime}$, with $\left(i^{\prime}, j^{\prime}\right)$ entry 1 and $(i, j)$ entry 0 .

Let the sequence of matrices generated by the algorithm be $B_{0}, B_{1}, B_{2}, \ldots, B_{s}$. Clearly $B_{i} \in \mathcal{H}(m, n)$ for $i=1,2, \ldots, s$. We claim that per $B_{i} \geq$ per $B_{i-1}$ for $i=1,2, \ldots, s$. To see this let $C=B_{i}$ and $D=B_{i-1}$. Since $D$ is fully indecomposable, Lemma 2.2 implies that $d_{i, i+1}=1(i=1,2, \ldots, n-1)$ and $d_{11}=d_{n n}=1$. First assume that $D$ has an interchangeable column pair. Let $(k, l)$ and $(k-1, l)$ be the interchangeable column pair chosen to construct $C$. If $d_{k k}=0$, then (by the choice of $l$ ) $a_{j k}=0$ for $j>k$. This would imply that column $k$ of $D$ has just one nonzero entry, contrary to the full indecomposability of $D$. Hence $d_{k k}=1$. By Lemma 2.2,

$$
\text { per } D(k, l)=\text { per } D[\langle 1, l-1\rangle] \text { per } D[\langle k+1, n\rangle]
$$

and

$$
\text { per } D(k-1, l)=\text { per } D[\langle 1, l-1\rangle] \text { per } D[\langle k, n\rangle] \text {. }
$$

The first factors in the righthand sides of (2) and (3) are the same. In the second factors, note that $D[\langle k+1, n\rangle]$ is a principal submatrix of $D[\langle k, n\rangle]$. Since $d_{k k}=1$, 
per $D[\langle k+1, n\rangle] \leq$ per $D[\langle k, n\rangle]$. Thus, by (2) and $(3)$, per $D(k, l) \leq$ per $D(k-1, l)$. By expanding per $C$ and per $D$ about column $l$ and noting that

$$
\text { per } C(k-1, l)=\operatorname{per} D(k-1, l) \text {, }
$$

the previous inequality gives per $D \leq$ per $C$. As $C, D \in \mathcal{H}(m, n)$ and per $D=P(m, n)$, it follows that per $C=P(m, n)=$ per $D$. A similar argument shows that if $C$ is obtained from $D$ by an interchangeable row pair, then per $C=\operatorname{per} D$.

Thus, $B_{s} \in \mathcal{H}(m, n)$ and per $B_{s}=P(m, n)$. Either $B_{s}$ is partly decomposable, or $B_{s}$ is fully indecomposable and has no interchangeable pairs. In the former case, apply induction to each fully indecomposable component of $B_{s}$ to arrive at a matrix in $\mathcal{H}(m, n)$ of maximum permanent, with each fully indecomposable component staircased. In the latter case $B_{s}$ is staircased, and hence each of its fully indecomposable components (of which there is only 1 ) is staircased.

We conclude this section with a theorem that gives a restriction on the staircased structure of each $A_{i}$ of a matrix $A$ in form (1) with maximum permanent.

Lemma 2.4 Let $A$ be an n-by-n fully indecomposable staircased Hessenberg (0,1)-matrix. Then

$$
\frac{2}{3} \geq \frac{\operatorname{per} A(n)}{\operatorname{per} A} \geq \frac{1}{2}
$$

Proof. Note that

$$
\text { per } A=\operatorname{per} A(n)+\text { per } A(n-1, n) \text {. }
$$

Since $A$ is staircased $A(n-1, n) \leq A(n)$ (entrywise). Hence per $A(n-1, n) \leq$ per $A(n)$, and per $A \leq 2$ per $A(n)$. This shows that per $A(n) /$ per $A \geq 1 / 2$.

For the other inequality note that since $A$ is a fully indecomposable staircased Hessenberg matrix, so is $A(n)$. Thus, by the above inequality, per $A(\langle n-1, n\rangle) \geq$ per $A(n) / 2$. By expansion along the last row

$$
\text { per } \begin{aligned}
A & \geq \operatorname{per} A(n)+\operatorname{per} A(\langle n-1, n\rangle) \\
& \geq \operatorname{per} A(n)+\operatorname{per} A(n) / 2 \\
& =3 \operatorname{per} A(n) / 2
\end{aligned}
$$

It follows that $2 / 3 \geq$ per $A(n) / \operatorname{per} A$.

Observe that $A(n)$ can be replaced by $A(1)$ in the above proposition.

Theorem 2.5 Let $A$ be an n-by-n fully indecomposable staircased Hessenberg $(0,1)$-matrix. Assume that $i, j, k$ are positive integers such that $a_{i j}$ is the first 1 in the ith row of $A$, $a_{i+1, k+1}$ is the first 1 in the $(i+1)$ st row of $A$, and $k-j \geq 2$. Let $B$ be the matrix obtained from $A$ by replacing its $(i, j)$-entry by 0 and its $(i+1, k)$-entry by 1 . Then $B$ is a Hessenberg $(0,1)$-matrix and per $B>\operatorname{per} A$. 
Proof. Let $C$ be the matrix obtained from $A$ by replacing $a_{i j}$ by 0 . Since $A$ is fully indecomposable and staircased, so is $C$. Also by Lemma 2.2(c)

$$
\text { per } C(i, j)=\operatorname{per} C[\langle 1, j-1\rangle] \text { per } C[\langle i+1, n\rangle]
$$

and

$$
\text { per } C(i+1, k)=\text { per } C[\langle 1, k-1\rangle] \text { per } C[\langle i+2, n\rangle] \text {. }
$$

By Lemma 2.4, per $C[\langle i+2, n\rangle] \geq$ per $C[\langle i+1, n\rangle] / 2$, and by repeated application of the preceding proposition

$$
\text { per } C[\langle 1, k-1\rangle] \geq(3 / 2) \text { per } C[\langle 1, k-2\rangle] \geq \cdots \geq(3 / 2)^{k-j} \text { per } C[\langle 1, j-1\rangle] \text {. }
$$

Substituting these bounds into (5) and using (4) and $k-j \geq 2$,

$$
\text { per } C(i+1, k) \geq(9 / 8) \operatorname{per} C(i, j)>\text { per } C(i, j) \text {. }
$$

Since per $A=$ per $C+$ per $C(i, j)$ and per $B=$ per $C+$ per $C(i+1, k)$, it follows that per $B>$ per $A$.

Note that Theorem 2.5 implies that if $A \in \mathcal{H}(m, n)$, each fully indecomposable component of $A$ is in staircased form and per $A$ is maximal, then no "step" of zeros has width 3 or more; that is, $\sum_{i=1}^{r}\left(a_{r i}-a_{r+1, i}\right) \leq 2$ for $r=1,2, \ldots, n-1$. Note that the bound is tight as $P(11,4)=6$ is achieved by the following matrix with a step of zeros of width 2 (i.e., $\left.\sum_{i=1}^{3}\left(a_{3 i}-a_{4 i}\right)=2\right)$ :

$$
A=\left[\begin{array}{llll}
1 & 1 & 0 & 0 \\
1 & 1 & 1 & 0 \\
1 & 1 & 1 & 1 \\
0 & 0 & 1 & 1
\end{array}\right]
$$

\section{$3 \quad P(m, n)$ for $n \leq m \leq(7 n-1) / 3$}

Clearly $P(m, n)=0$ for $m<n$. For $n \leq m \leq 2 n$, Brualdi, Goldwasser and Michael [BGM, Theorem 2.2] show that for an $n$-by- $n(0,1)$-matrix with $m$ entries equal to 1 , the maximum permanent is $2^{\lfloor(m-n) / 2\rfloor}$. Additionally, they characterize the matrices achieving the maximum. The following proposition follows from their characterization by noting that each matrix achieving the maximum is combinatorially equivalent to a Hessenberg matrix. We give a self-contained proof here that makes use of the matrices being Hessenberg. Let $H_{n}=\left[h_{i j}\right]$ be the $n$-by- $n$ Hessenberg matrix with $h_{i j}=1$ if $j \leq i+1$. Note that per $H_{n}=2^{n-1}$ and $\operatorname{nnz}\left(H_{n}\right)=\left(n^{2}+3 n-2\right) / 2$.

Theorem 3.1 For integers $m$ and $n$ with $2 \leq n \leq m \leq 2 n$,

$$
P(m, n)=2^{\lfloor(m-n) / 2\rfloor} .
$$


Proof. Let $t=\lfloor(m-n) / 2\rfloor$, and let $A$ be the direct sum of $t \geq 0$ matrices $H_{2}$ and $n-2 t \geq 0$ matrices $H_{1}$. Then $A$ is $n$-by- $n, \operatorname{nnz}(A)=4 t+n-2 t=n+2 t$, and per $A=2^{t}$. If $m-n$ is even, then nnz $A=m$, and per $A=2^{t}$. If $m-n$ is odd, then the matrix $A^{\prime}$ obtained from $A$ by replacing the 0 in its $(n, 1)$ position by a 1 has $m$ nonzeros and permanent $2^{t}$. Hence, $P(m, n) \geq 2^{\lfloor(m-n) / 2\rfloor}$.

The proof that $P(m, n) \leq 2^{\lfloor(m-n) / 2\rfloor}$ is by induction on $m$. If $m=n$, then $P(n, n)$ is the largest permanent of an $n$-by- $n(0,1)$-matrix with $n$ entries equal to 1 , and this is clearly at most $1=2^{0}=2^{\lfloor(m-n) / 2\rfloor}$, as desired. Assume that $m>n$, and proceed by induction. Let $A$ be an $n$-by- $n$ Hessenberg $(0,1)$-matrix with $\operatorname{nnz}(A)=m$. By Theorem 2.3, assume that $A$ has the form (1), where $A_{i}$ is a fully indecomposable staircased $n_{i^{-}}$ by- $n_{i}$ Hessenberg matrix for $i=1, \ldots, b$. If each $n_{i}=1$, then per $A=1 \leq 2^{\lfloor(m-n) / 2\rfloor}$. Otherwise, there exists an $i$ such that $n_{i} \geq 2$. Since $A_{i}$ is staircased and Hessenberg, the observation after Lemma 2.4 implies that

$$
\text { per } A_{i} \leq 2 \text { per } A_{i}(1) \text {. }
$$

Let $j$ be the row of $A$ that intersects the first row of $A_{i}$. Then (6) implies that per $A \leq$ 2 per $A(j)$. Note that nnz $(A(j)) \leq n n z(A)-3=m-3$. Hence, by induction, per $A(j) \leq$ $2^{\lfloor(m-3-(n-1)) / 2\rfloor}$. It follows that per $A \leq 2^{\lfloor(m-n) / 2\rfloor}$, and hence that $P(m, n) \leq 2^{\lfloor(m-n) / 2\rfloor}$, as desired.

Letting $E_{i j}$ be the matrix with $(i, j)$-entry equal to 1 and all other entries zero, the complete results for $n=2$ are given by the above theorem as:

$P(2,2)=1$, with equality for $A=H_{1} \oplus H_{1}$;

$P(3,2)=1$, with equality for $A=\left(H_{1} \oplus H_{1}\right)+E_{21}$;

$P(4,2)=2$, with equality for $A=H_{2}$.

For $n \geq 5$ and a subset of values of $m$ with $2 n+1 \leq m \leq(7 n-1) / 3$, the following recursion leads to an explicit formula for $P(m, n)$. In the next two results, we write $m=2 n+t ;$ thus $n \geq 3 t+1$ implies that $m \leq(7 n-1) / 3$.

Theorem 3.2 Let $t$ and $n$ be positive integers with $n \geq \max \{5,3 t+1\}$. Then

$$
P(2 n+t, n)=2 P(2(n-2)+t, n-2) .
$$

Proof. The assumptions on $t$ and $n$ imply that $2(n-2)+t \leq \frac{(n-2)^{2}+3(n-2)-2}{2}$, and hence $\mathcal{H}(2(n-2)+t, n-2) \neq \emptyset$. Let $A \in \mathcal{H}(2(n-2)+t, n-2)$ with per $A=P(2(n-2)+t, n-2)$. Then $H_{2} \oplus A \in \mathcal{H}(2 n+t, n)$ and has permanent $2 P(2(n-2)+t, n-2)$. Hence $P(2 n+t, n) \geq 2 P(2(n-2)+t, n-2)$.

We now prove that $P(2 n+t, n) \leq 2 P(2(n-2)+t, n-2)$. By Theorem 2.3 , there is a matrix $A \in \mathcal{H}(2 n+t, n)$ with permanent $P(2 n+t, n)$ of the form (1) with each fully indecomposable component a staircased, Hessenberg $(0,1)$-matrix. Let the order of $A_{i}$ be $n_{i}(i=1,2, \ldots, b)$. If some $n_{i}=2$, then $A_{i}=H_{2}$, and per $A=2$ per $A^{\prime}$, where $A^{\prime}$ is the matrix obtained from $A$ by deleting the rows and columns that intersect $A_{i}$. Since $\operatorname{nnz}\left(A^{\prime}\right) \leq \operatorname{nnz}(A)-4$, per $A^{\prime} \leq P(2(n-2)+t, n-2)$, and hence per $A \leq$ $2 P(2(n-2)+t, n-2)$, as desired. 
Suppose that $n_{i} \geq 3$ for $i=1, \ldots, b$. Then

$$
n=\sum_{i=1}^{b} n_{i} \geq 3 b .
$$

Also, by Lemma $2.2(\mathrm{a})$ and $(\mathrm{b}), \operatorname{nnz}\left(A_{i}\right) \geq 3 n_{i}-2(i=1,2, \ldots, b)$. Hence

$$
2 n+t=\operatorname{nnz}(A) \geq \sum_{i=1}^{b} \operatorname{nnz}\left(A_{i}\right) \geq 3 n-2 b,
$$

and thus $t \geq n-2 b$. This and (7) imply that $t \geq b$ and $3 t \geq n$, contradicting the hypothesis of the theorem.

Finally, suppose that some $n_{i}=1$, and some $n_{j} \geq 3$. Since $A_{j}$ is staircased, per $A_{j} \leq 2$ per $A_{j}(1)$. Hence

$$
\operatorname{per}\left(A_{i} \oplus A_{j}\right) \leq \operatorname{per}\left(H_{2} \oplus A_{j}(1)\right) .
$$

Let $A^{\prime}$ be the matrix obtained from $A$ by replacing the blocks $A_{i}$ and $A_{j}$ by $H_{2}$ and $A_{j}(1)$. Then $n n z\left(A^{\prime}\right) \leq \operatorname{nnz}(A)$, and per $A^{\prime} \geq$ per $A$. It follows that $A^{\prime}$ can be used rather than $A$. But $A^{\prime}$ has a 2-by-2 fully indecomposable block. This leads back to a case already considered. Hence $P(2 n+t, n) \leq 2 P(2(n-2)+t, n-2)$.

Corollary 3.3 Let $t$ be a positive integer. There exist constants $e_{t}$ and $o_{t}$ such that for all $n \geq \max \{5,3 t+1\}$

$$
P(2 n+t, n)=\left\{\begin{array}{cl}
e_{t} 2^{n / 2} & \text { if } n \text { is even } \\
o_{t} 2^{(n-1) / 2} & \text { if } n \text { is odd }
\end{array}\right.
$$

Proof. Theorem 3.2 shows that for $n \geq \max \{5,3 t+1\}$, the function $P(2 n+t, n)$ grows by a factor of 2 each time $n$ is increased by 2 . Thus, only the initial conditions need to be determined to have an exact formula for $P(2 n+t, n)$.

In particular, for $t=1$, take the initial conditions to be $e_{1}=P(9,4) / 4$ which is equal to 1 , and $o_{1}=P(7,3) / 2$ which is equal to $3 / 2$. An induction argument (using Theorem 3.2) can be given to show that $P(2 n+1, n)=e_{1} 2^{n / 2}$ if $n$ is even and $n \geq 5$, and $P(2 n+1, n)=o_{1} 2^{(n-1) / 2}$ if $n$ is odd and $n \geq 5$.

For $t \geq 2$, the initial conditions are obtained by setting

$$
e_{t}=\left\{\begin{array}{cl}
P(7 t, 3 t) / 2^{3 t / 2} & \text { if } t \text { is even } \\
P(7 t-2,3 t-1) / 2^{(3 t-1) / 2} & \text { if } t \text { is odd }
\end{array}\right.
$$

and

$$
o_{t}=\left\{\begin{array}{cl}
P(7 t, 3 t) / 2^{(3 t-1) / 2} & \text { if } t \text { is odd } \\
P(7 t-2,3 t-1) / 2^{(3 t-2) / 2} & \text { if } t \text { is even. }
\end{array}\right.
$$

Again, an induction argument can be used to show that the desired formula for $P(2 n+t, n)$ holds for $n \geq 3 t+1$.

In the next section, these constants $e_{t}$ and $o_{t}$ are explicitly determined. 


\section{$4 \quad P(m, n)$ for $2 n+1 \leq m \leq 8 n / 3$}

In this section we determine the exact values of $P(m, n)$ for $2 n+1 \leq m \leq 8 n / 3$. For $n \leq 2$ and $m$ in this range, $\mathcal{H}(m, n)=\emptyset$. Thus, we take $n \geq 3$. Denote by $T_{n}=\left[t_{i j}\right]$ the $n$-by- $n$ tridiagonal matrix with $t_{i j}=1$ if $|i-j| \leq 1$. Since per $T_{1}=1$, per $T_{2}=2$ and per $T_{n}=$ per $T_{n-1}+$ per $T_{n-2}$ for $n \geq 3$, it follows that per $T_{n}$ equals the $n$-th Fibonacci number, $f_{n}$.

We begin by establishing lower bounds on $P(m, n)$. Note that for fixed $n, P(m, n)$ is a nondecreasing function of $m$. For integers $m$ and $n$ with $2 n+1 \leq m \leq 8 n / 3$, define

$$
u(m, n)=\left\{\begin{array}{cl}
2^{m / 4}, & \text { if } m \equiv 0 \bmod 4 \\
2^{(m-1) / 4}, & \text { if } m \equiv 1 \bmod 4 \\
\frac{5}{4} \times 2^{(m-2) / 4}, & \text { if } m \equiv 2 \bmod 4 \\
\frac{3}{2} \times 2^{(m-3) / 4}, & \text { if } m \equiv 3 \bmod 4 .
\end{array}\right.
$$

Proposition 4.1 If $m$ and $n$ are positive integers with $2 n+1 \leq m \leq 8 n / 3$, then $P(m, n) \geq u(m, n)$.

Proof. Let $m \geq 2 n+1$ and first suppose that $m \equiv 0 \bmod 4$. Let $r=(m-2 n) / 2$ and $s=(8 n-3 m) / 4$. Then $r \geq 1$ and $s \geq 0$ are integers. Define $A$ to be the direct sum of $r$ matrices $H_{3}$ and $s$ matrices $H_{2}$. Then $\operatorname{nnz}(A)=m, A$ is $n$-by- $n$ and per $A=2^{2 r+s}=$ $2^{m / 4}=u(m, n)$. Hence $P(m, n) \geq u(m, n)$.

Second suppose that $m \equiv 1 \bmod 4$, and thus $3 m \leq 8 n-1$. Since $m \equiv 1 \bmod 4$, $u(m, n)=u(m-1, n)$. Clearly, $P(m, n) \geq P(m-1, n)$. By the previous case $P(m-1, n) \geq$ $u(m-1, n)$. Hence, $P(m, n) \geq u(m-1, n)=u(m, n)$.

Now suppose that $m \equiv 2 \bmod 4$, and thus $3 m \leq 8 n-2$. Since $2 n+1 \leq m \leq 8 n / 3$, it follows that $n \geq 4$ and $m \geq 10$. Set $r=(m-2-2 n) / 2$ and $s=(8 n-3 m-2) / 4$. Then $r$ and $s$ are nonnegative integers. Define $A$ to be the direct sum of $r$ matrices $H_{3}, s$ matrices $H_{2}$ and one $T_{4}$. Then $\operatorname{nnz}(A)=m, A$ is $n$-by- $n$ and per $A=5 \times 2^{2 r+s}=\frac{5}{4} \times 2^{(m-2) / 4}=u(m, n)$. Hence $P(m, n) \geq u(m, n)$.

Finally suppose that $m \equiv 3 \bmod 4$, and thus $3 m \leq 8 n-3$. Let $r=(m-1-2 n) / 2$ and $s=(8 n-3 m-3) / 4$. Then $r$ and $s$ are nonnegative integers. Let $A$ be the direct sum of $r$ matrices $H_{3}, s$ matrices $H_{2}$ and one $T_{3}$. Then $\operatorname{nnz}(A)=m, A$ is $n$-by- $n$ and per $A=3 \times 2^{2 r+s}=\frac{3}{2} \times 2^{(m-3) / 4}=u(m, n)$. Hence $P(m, n) \geq u(m, n)$.

The main result of this section is that $P(m, n)=u(m, n)$ for $m \leq 8 n / 3$. The proof of the main result requires several preliminary lemmas. Recall that $E_{i j}$ is a matrix with $(i, j)$-entry equal to 1 and all other entries 0 .

Lemma 4.2 Let $k, l$ and $p$ be integers with $k \geq 2, l \geq 1$ and $p \geq 1$. Let $B$ be $a p$-by- $p$ Hessenberg $(0,1)$-matrix, and $x$ a $(0,1)$-vector that is entrywise less than or equal to the 
first column of $B$. Let $R$ and $S$ be the partitioned Hessenberg matrices

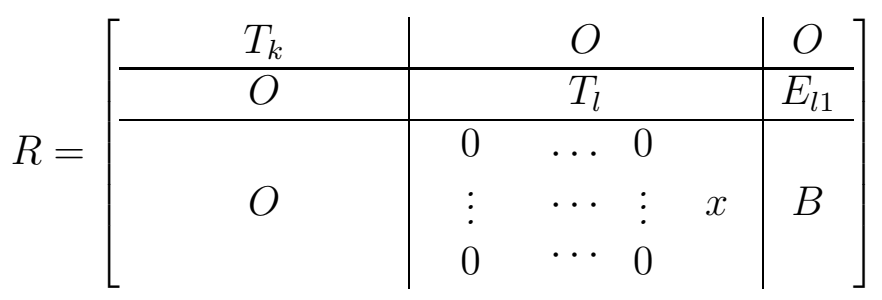

and

$$
S=\left[\begin{array}{c|c|c}
T_{k} & E_{k 1} & O \\
\hline E_{1, k-1}+E_{1 k} & T_{l} & O \\
\hline O & O & B
\end{array}\right] .
$$

Then per $S \geq$ per $R$, and the fully indecomposable components of $S$ are $S[\langle 1, k+l\rangle]$ and those of $B$.

Proof. Since every transversal of $S$ that contains the $(k, k+1)$-entry contains either the $(k+1, k-1)$ - or $(k+1, k)$-entry,

per $S=\operatorname{per} T_{k}$ per $T_{l}$ per $B+\left[\operatorname{per} T_{k}(k, k)+\operatorname{per} T_{k}(k, k-1)\right]$ per $T_{l}(1)$ per $B$ $=$ per $T_{k}$ per $T_{l}$ per $B+$ per $T_{k} f_{l-1}$ per $B$.

Since every transversal of $R$ containing the $(k+l, k+l+1)$-entry contains a 1 of $x$,

$$
\text { per } \begin{aligned}
R & =\operatorname{per} T_{k} \text { per } T_{l} \text { per } B+\operatorname{per} T_{k} \text { per } T_{l}(l) \text { per } B^{\prime} \\
& =\operatorname{per} T_{k} \text { per } T_{l} \text { per } B+\operatorname{per} T_{k} f_{l-1} \text { per } B^{\prime},
\end{aligned}
$$

where $B^{\prime}$ is the matrix obtained from $B$ by replacing the first column of $B$ with $x$. Since $x$ is entrywise less than or equal to the first column of $B, \mathrm{~m}$ per $B^{\prime} \leq$ per $B$. Thus,

$$
\text { per } R \leq \text { per } T_{k} \text { per } T_{l} \text { per } B+\text { per } T_{k} f_{l-1} \text { per } B=\text { per } S \text {. }
$$

It is easy to verify that $S[\langle 1, k+l\rangle]$ is fully indecomposable and that $S=S[\langle 1, k+$ $l\rangle] \oplus B$. Hence the fully indecomposable components of $S$ are $S[\langle 1, k+l\rangle]$ and the fully indecomposable components of $B$.

Considering $P R^{T} P$ and $P S^{T} P$, where $P$ is the reverse permutation matrix, the following result is obtained.

Lemma 4.3 Let $r, s$ and $q$ be integers with $r \geq 1, s \geq 2$ and $q \geq 1$. Let $C$ be a $q$-by-q Hessenberg $(0,1)$-matrix, and $y^{T}$ a $(0,1)$-vector that is entrywise less than or equal to the 
last row of $C$. Let $U$ and $V$ be the partitioned Hessenberg matrices

$$
U=\left[\begin{array}{ccc|c|c}
C & E_{q 1} & O \\
\hline y^{T} & & & \\
0 & \cdots & 0 & T_{r} & O \\
\vdots & \vdots & \vdots & & \\
0 & \cdots & 0 & & T_{s}
\end{array}\right]
$$

and

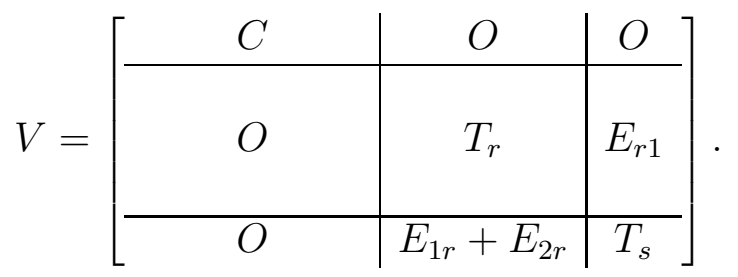

Then per $V \geq$ per $U$, and the fully indecomposable components of $V$ are $V[\langle q+1, q+r+s\rangle]$ and those of $C$.

Let $m$ and $n$ be integers with $2 n+1 \leq m \leq 8 n / 3$. Define $\mathcal{S}(m, n)$ to be the set of all $n$-by- $n(0,1)$-matrices $A$ such that $\operatorname{nnz}(A) \leq m$, per $A=P(m, n), A$ has form (1) where each $A_{i}$ is a fully indecomposable staircased Hessenberg matrix and each $A_{i j}=0$ for $i \neq j$.

By Theorem 2.3, $\mathcal{S}(m, n) \neq \emptyset$. Since $P(m, n)$ is a nondecreasing function of $m$, if $\widehat{A}$ is an $n$-by- $n$ matrix that is a direct sum of fully indecomposable staircased Hessenberg matrices with $\operatorname{nnz}(\widehat{A}) \leq m$ and per $\widehat{A} \geq P(m, n)$, then per $\widehat{A}=P(m, n)$ and $\widehat{A} \in \mathcal{S}(m, n)$. In particular, if $A \in \mathcal{S}(m, n)$ and some direct sum of fully indecomposable components of $A$ has the form $R$ described in Lemma 4.2 with $\mathrm{nnz}(x) \geq 2$, then the matrix $A^{\prime}$ obtained from $A$ by replacing $R$ by the matrix $S$ of Lemma 4.2 necessarily belongs to $\mathcal{S}(m, n)$. A similar statement holds for a matrix $A^{\prime \prime}$ obtained from $A \in \mathcal{S}(m, n)$ by replacing a direct sum of fully indecomposable components of the form $U$, as in Lemma 4.3, by the matrix $V$ of Lemma 4.3 .

The next lemma shows that if $m \leq 8 n / 3$, then $\mathcal{S}(m, n)$ contains a matrix in one of the following four special forms.

Lemma 4.4 For all positive integers $m$ and $n$ with $2 n+1 \leq m \leq 8 n / 3$, there is a matrix $A \in \mathcal{S}(m, n)$ with at least one of the following properties:

(a) $A_{i}=T_{n_{i}}$ for all $i$;

(b) $A_{i} \notin\left\{T_{2}, T_{3}, T_{4}\right\}$ for all $i$;

(c) $A_{i}=H_{3}$ for at least one $i$;

(d) $A_{i}=T_{3}$ for at least one $i$. 
Proof. Suppose on the contrary that none of these statements hold. Then for every $A \in \mathcal{S}(m, n)$, at least one of its fully indecomposable components is not tridiagonal, at least one of its fully indecomposable components is $T_{2}$ or $T_{4}$, and none of its fully indecomposable components has order 3 .

Case 1: There is an $A \in \mathcal{S}(m, n)$ each of whose fully indecomposable components has order at least 4.

Among all such matrices $A$, choose one with the minimum number of fully indecomposable components equal to $T_{4}$. By assumption there is an $i$ with $A_{i}=T_{4}$, and a $j$ such that $A_{j} \neq T_{n_{j}}$ and $n_{j} \geq 4$. Let $R=A_{i} \oplus A_{j}$, and let $l$ be the first index such that column $l$ of $A_{j}$ does not equal column $l$ of $T_{n_{j}}$. Then $A_{i} \oplus A_{j}$ has the form $R$ of Lemma 4.2 with $k=4$ and $\operatorname{nnz}(x) \geq 2$. Note that $1 \leq l \leq n_{j}-2$, and thus the order of $B$ is $p \geq 2$. Since $A_{j}$ is fully indecomposable and staircased, so is $B$. Define $S$ as in Lemma 4.2, and let $A^{\prime}$ be the matrix obtained from $A$ by replacing $R$ by $S$. Since $n n z(x) \geq 2, \operatorname{nnz}\left(A^{\prime}\right) \leq \operatorname{nnz}(A)$. By Lemma 4.2 , per $A^{\prime} \geq$ per $A$. Hence $A^{\prime} \in \mathcal{S}(m, n)$. The fully indecomposable components of $S$ are one of order $4+l$ and $B$. The choice of $A$ requires that $p \leq 4$ (else every component of $A^{\prime}$ has order at least 4 and $A^{\prime}$ has fewer fully indecomposable components equal to $\left.T_{4}\right)$.

First suppose $p=2$. Then $l=n_{j}-2$ and $A_{j}=T_{n_{j}}+E_{n_{j}, n_{j}-2}$. Let $\widehat{A}$ be obtained from $A$ by interchanging $A_{i}$ and $A_{j}$, and consider $U=A_{j} \oplus A_{i}$. Then

$$
U=\left[\begin{array}{ccccc|c|cc} 
& & & & & 0 & & \\
& & & & & & & \\
& & & & & & \\
& & & & & 0 & & \\
& & & & & & & \\
\hline 0 & \cdots & 0 & 1 & 1 & 1 & 0 & 0 \\
\hline & & O & & & O & T_{4}
\end{array}\right],
$$

Define $V$ as in Lemma 4.3, and let $A^{\prime \prime} \in \mathcal{S}(m, n)$ be the matrix obtained from $\widehat{A}$ by replacing $U$ by $V$. The fully indecomposable components of $V$ are $T_{n_{j}-1}$ and a matrix of order 5 . Because $n_{j} \geq 4$, none of the fully indecomposable components of $A^{\prime \prime}$ has order 1 or 2. Since neither (c) nor (d) holds, $A^{\prime \prime}$ has no fully indecomposable component of order 3. Hence, each fully indecomposable component of $A^{\prime \prime}$ has order at least 4 . By the choice of $A, A^{\prime \prime}$ has at least as many fully indecomposable components equal to $T_{4}$ as $A$, and thus $n_{j}=5$. Hence $A_{i} \oplus A_{j}=T_{4} \oplus\left(T_{5}+E_{53}\right)$ is 9-by-9 with 24 entries equal to 1 and permanent $5 \times 10=50$. The matrix $H_{3} \oplus H_{3} \oplus H_{3}$ is 9-by-9 with 24 entries equal to 1 and permanent $4^{3}$. Thus replacing $A_{i} \oplus A_{j}$ in $A$ by $H_{3} \oplus H_{3} \oplus H_{3}$ results in a Hessenberg $(0,1)$-matrix with the same number of ones as $A$, but larger permanent. This is impossible, since per $A=P(m, n)$. We conclude that $p \neq 2$.

Next suppose that $p=3$. Then $B \in\left\{T_{3}, H_{3}\right\}$, and hence $A^{\prime}$ has either $T_{3}$ or $H_{3}$ as a fully indecomposable component contrary to the assumption that neither (c) nor (d) holds.

Thus $p=4$. The fully indecomposable components of $S$ are one of order at least 5 , and $B$ of order 4 . Thus all fully indecomposable components of $A^{\prime}$ have order at least 4 . 
If $B \neq T_{4}$, then we are led to the contradiction that $A^{\prime}$ has fewer fully indecomposable components equal to $T_{4}$ than $A$. Thus $B=T_{4}$. Since $A_{j}$ is staircased and $B=T_{4}$, the definition of $l$ implies that $A_{j}=T_{n_{j}}+E_{n_{j}-2, n_{j}-4}$. Now $U=A_{j} \oplus A_{i}$ has the form of $U$ in Lemma 4.3 with $r=3, s=4, C=T_{q}$ and $y^{T}=\left[\begin{array}{lllll}0 & \cdots & 0 & 1 & 1\end{array}\right]$. Using Lemma 4.3, replace $U$ in $A$ by $V$ to obtain a matrix $A^{\prime \prime \prime} \in \mathcal{S}(m, n)$. Arguing as with $p=2$, by the choice of $A$ the matrix $V$ must have a fully indecomposable component of order 4 ; thus $q=4$. Hence $n_{j}=7$ and $A_{j}=T_{7}+E_{53}$. It follows that

$$
\operatorname{per}\left(A_{i} \oplus A_{j}\right)=5 \times(21+4)=125<126=6 \times 21=\operatorname{per}\left(\left(A_{i}+E_{31}\right) \oplus T_{7}\right) .
$$

Replacing $A_{i}$ with $A_{i}+E_{31}$ and $A_{j}$ with $T_{7}$ gives a matrix with the same number of nonzero entries as $A$ but with a larger permanent. Therefore, Case 1 leads to a contradiction.

Case 2: Every $A \in \mathcal{S}(m, n)$ has at least one fully indecomposable component of order less than 4 .

Among the matrices in $\mathcal{S}(m, n)$, choose $A$ to have the minimum number of fully indecomposable components of order 1 . We claim that $A$ has no fully indecomposable component of order 1. Suppose on the contrary that some $n_{i}$ equals 1 . Since neither (a) nor (c) holds, there is a $j$ such that $A_{j} \notin\left\{H_{3}, T_{n_{j}}\right\}$. In particular, $n_{j} \geq 4$. Since $A_{j}$ is staircased, (6) implies that per $A_{j} \leq 2$ per $A_{j}(1)$, and thus $\operatorname{per}\left(A_{i} \oplus A_{j}\right) \leq \operatorname{per}\left(H_{2} \oplus A_{j}(1)\right)$. Hence, replacing $A_{i}$ and $A_{j}$ by $H_{2}$ and $A_{j}(1)$, respectively, results in a matrix $A^{\prime} \in \mathcal{S}(m, n)$. However, $A^{\prime}$ has one less fully indecomposable component of order 1 , contrary to the choice of $A$. Therefore, $A$ has no fully indecomposable component of order 1 .

Among the matrices in $\mathcal{S}(m, n)$ with no fully indecomposable component of order 1 , now choose $A$ to have the minimum number of fully indecomposable components of order 2. We claim that $A$ has no fully indecomposable component of order 2. Suppose on the contrary that $n_{i}$ equals 2 . Since neither (a) nor (c) holds, there is a fully indecomposable component $A_{j}$ of order at least 4 that is not $T_{n_{j}}$. Let $R=A_{i} \oplus A_{j}$, and let $l$ be the first index such that column $l$ of $A_{j}$ does not equal column $l$ of $T_{n_{j}}$. Then $A_{i} \oplus A_{j}$ has the form of $R$ in Lemma 4.2 with $k=2$ and $\operatorname{nnz}(x) \geq 2$. Using Lemma 4.2, replace $R$ by $S$ to obtain a matrix $A^{\prime} \in \mathcal{S}(m, n)$. The choice of $A$ requires that some fully indecomposable component of $S$ has order 2. Since the fully indecomposable components of $S$ are $B$ and a matrix of order $2+l, B$ must have order 2. Thus $A_{j}=T_{n_{j}}+E_{n_{j}, n_{j-2}}$. Let $\widehat{A}$ be obtained from $A$ by interchanging $A_{i}$ and $A_{j}$, and consider $U=A_{j} \oplus A_{i}$. Then

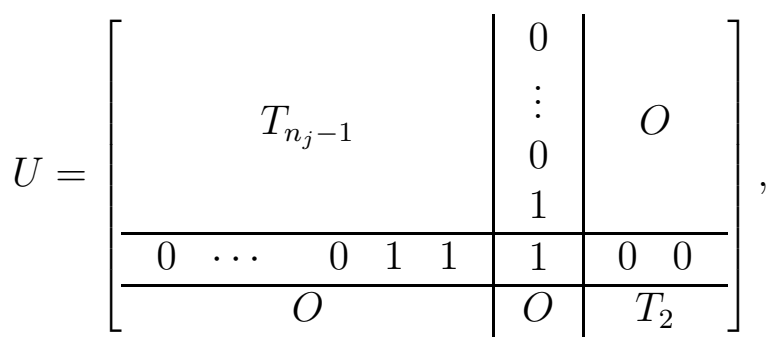

and the matrix $V$ in Lemma 4.3 is $T_{n_{j}-1} \oplus H_{3}$. Applying Lemma 4.3, replace $U$ in $\widehat{A}$ by $V$ to obtain a matrix $A^{\prime \prime} \in \mathcal{S}(m, n)$. But $A^{\prime \prime}$ has $H_{3}$ as a fully indecomposable component, 
contrary to our assumption that (c) does not hold. Thus, we are led to a contradiction, and conclude that there is an $A \in \mathcal{S}(m, n)$ with no fully indecomposable components of orders 1 or 2 . Since no fully indecomposable component of a matrix in $\mathcal{S}(m, n)$ has order 3 , Case 2 leads to a contradiction.

Both Cases 1 and 2 lead to a contradiction, thus our original supposition that none of (a)- $(d)$ hold is false.

In the next lemma, the bound $P(m, n) \leq u(m, n)$, with $u(m, n)$ as defined at the beginning of this section, is obtained in the case that $\mathcal{S}(m, n)$ contains a matrix of a special type.

Lemma 4.5 Let $m$ and $n$ be positive integers with $2 n+1 \leq m \leq 8 n / 3$. Suppose that there exists $A \in \mathcal{S}(m, n)$ such that $A_{i}=H_{n_{i}}$ for all $i$. Then

$$
\text { per } A \leq u(m, n) \text {. }
$$

Proof. Note that $\frac{\mathrm{nnz}\left(H_{k}\right)}{k}>8 / 3$ for $k \geq 4, \frac{\mathrm{nnz}\left(H_{3}\right)}{3}=8 / 3$ and $\frac{\mathrm{nnz}\left(H_{k}\right)}{k}<8 / 3$ for $k=1,2$. Since $\operatorname{nnz}(A) \leq 8 n / 3$, it follows that either $n_{i}=3$ for all $i$ or $n_{i} \leq 2$ for at least one $i$.

Note that per $\left(H_{a} \oplus H_{b}\right)=$ per $\left(H_{a+1} \oplus H_{b-1}\right)$ for all $a, b$ with $b \geq 2$. In particular, since $\operatorname{nnz}\left(H_{1} \oplus H_{b}\right) \geq \operatorname{nnz}\left(H_{2} \oplus H_{b-1}\right)$ for all $b \geq 2$, we can replace each occurence of $H_{1} \oplus H_{b}$ in $A$ by $H_{2} \oplus H_{b-1}$. Similarly, we can replace each $H_{2} \oplus H_{b}$ in $A$ by $H_{3} \oplus H_{b-1}$ for all $b \geq 3$. Therefore, without loss of generality we may assume that one of the following holds: (a) all fully indecomposable components of $A$ are matrices $H_{3}$, (b) $A$ has a fully indecomposable component of order 2 , and all other fully indecomposable components have orders 2 or 3, (c) $A$ has a fully indecomposable component of order 1 , and all other fully indecomposable components have orders 1 or 2 .

First suppose that (a) holds; say $A$ is the direct sum of $r$ matrices $H_{3}$. Then $n=3 r$, $\operatorname{nnz}(A)=8 r=8 n / 3$ and per $A=4^{r}=2^{2 r}$. It follows that $m=8 n / 3, m \equiv 0 \bmod 4$ and that per $A=2^{m / 4}=u(m, n)$, as desired.

Next suppose that (b) holds; say that $A$ is the direct sum of $s \geq 1$ matrices $H_{2}$ and $r \geq 0$ matrices $H_{3}$. Then $n=2 s+3 r, \operatorname{nnz}(A)=4 s+8 r$ and per $A=2^{s+2 r}=$ $2^{\mathrm{nnz}(A) / 4}$. Since $A$ has the maximum permanent of matrices in $\mathcal{S}(m, n)$, by Proposition $4.1 m \in\{\operatorname{nnz}(A), \operatorname{nnz}(A)+1\}$. Thus, since $\operatorname{nnz}(A)$ is a multiple of $4, m \equiv 0$ mod 4 or $m \equiv 1 \bmod 4$. If $m \equiv 0 \bmod 4$, then $m=\operatorname{nnz}(A)$ and per $A=2^{m / 4}=u(m, n)$, as desired. If $m \equiv 1 \bmod 4$, then $m=\operatorname{nnz}(A)+1$ and per $A=2^{(m-1) / 4}=u(m, n)$, as desired.

Finally, suppose that (c) holds; say that $A$ is the direct sum of $l \geq 1$ matrices $H_{1}$ and $s \geq 0$ matrices $H_{2}$. Then $n=l+2 s, \operatorname{nnz}(A)=l+4 s$ and per $A=2^{s}=2^{(\mathrm{nnz}(A)-n) / 2}$. Since $A$ has the maximum permanent of matrices in $\mathcal{S}(m, n)$, by Proposition $4.1 \mathrm{~m} \in$ $\{\operatorname{nnz}(A), \operatorname{nnz}(A)+1\}$. Thus $m \leq l+4 s+1 \leq 2 n$, so (c) cannot occur.

Next we use a result from the literature on arbitrary $(0,1)$-matrices to characterize equality for a bound on per $A$ for $A \in \mathcal{H}(m, n)$. This lemma is useful in proving that $P(m, n) \leq u(m, n)$ in the case that $\mathcal{S}(m, n)$ contains a matrix each of whose fully indecomposable components is tridiagonal. In the next proof, the $n$-by- $n$ "cycle matrix" $C_{n}=\left[c_{i j}\right]$ has $c_{i, i+1}=1$ for $1 \leq i \leq n-1, c_{n 1}=1$ and all other $c_{i j}=0$. 
Lemma 4.6 Let $A \in \mathcal{H}(m, n)$ be fully indecomposable and staircased. Then

$$
\text { per } A \leq 2^{m-2 n}+1
$$

with equality if and only if $A \in\left\{T_{2}, T_{3}, T_{4}\right\}$.

Proof. By [BR, Theorem 7.4.14] and the characterization by Foregger, (8) holds for an arbitrary $(0,1)$-matrix with equality if and only if $n \geq 2$ and there exist permutation matrices $P$ and $Q$ and a positive integer $p$ such that $P A Q$ has the form

$$
\left[\begin{array}{ccccc}
B_{1} & O & \cdots & O & E_{1} \\
E_{2} & B_{2} & \cdots & O & O \\
\vdots & \vdots & \ddots & \vdots & \vdots \\
O & O & \cdots & B_{p-1} & O \\
O & O & \cdots & E_{p} & B_{p}
\end{array}\right]
$$

where $B_{i}$ is $n_{i}$-by- $n_{i}, B_{i}=I+C_{n_{i}}$ if $n_{i} \geq 2, B_{i}=[1]$ if $n_{i}=1$, and $\operatorname{nnz}\left(E_{i}\right)=1$ $(i=1,2, \ldots, p)$.

Since $A$ is staircased and fully indecomposable, $3 n-2 \leq m$ by Lemma 2.2 (a) and (b). Let $q$ be the number of $n_{i}$ that are equal to 1 . Then $3 n-2 \leq m=2 n+p-q$, giving $n+q \leq p+2$. Also $2 p-q \leq \sum_{i=1}^{p} n_{i}=n$. Adding these two inequalities gives $p \leq 2$ and thus $n+q \leq 4$.

The proof is completed by making the following observations. If $n=4$, then necessarily $q=0, p=2$ and $A=T_{4}$. If $n=2$, then since $A$ is fully indecomposable Hessenberg, $A=T_{2}$. If $n=3$, then since $A$ is fully indecomposable Hessenberg, $A=H_{3}$ or $A=T_{3}$. It is easy to verify that equality does not hold in (8) when $A=H_{3}$.

Using the above lemmas, we now determine an upper bound on $P(m, n)$.

Theorem 4.7 If $m$ and $n$ are positive integers with $2 n+1 \leq m \leq 8 n / 3$, then $P(m, n) \leq$ $u(m, n)$.

Proof. The proof is by induction on $n$. It is easy to verify that $P(7,3)=3$ and $P(8,3)=4$, thus for $n=3, P(m, n)=u(m, n)$.

Assume that $n \geq 4$. It suffices to show that for some (and hence every) matrix $A \in \mathcal{S}(m, n)$, we have per $A \leq u(m, n)$. Note, by Lemma 4.4 , we can assume that at least one of $(a)$ - $(d)$ holds. Also, by Lemma 4.5 , if there is an $A \in \mathcal{S}(m, n)$ each of whose fully indecomposable components is an $H_{n_{i}}$, then $P(m, n) \leq u(m, n)$. Henceforth we assume that every matrix in $\mathcal{S}(m, n)$ has at least one fully indecomposable component $A_{i}$ with $A_{i} \neq H_{n_{i}}$. In particular, this implies that $\operatorname{nnz}(A)=m$ for each $A \in \mathcal{S}(m, n)$, since if $\operatorname{nnz}(A)<m$, then a 0 in this fully indecomposable component could be changed to 1 , increasing the permanent.

Case 1: Statement (a) of Lemma 4.4 holds. 
Then there is an $A \in \mathcal{S}(m, n)$ so that $A_{i}=T_{n_{i}}$ for all $i$. Each row of the chart below gives a Hessenberg $(0,1)$-matrix $W$ and a Hessenberg $(0,1)$-matrix $X$ such that $W$ and $X$ have the same order, $\operatorname{nnz}(W) \geq \operatorname{nnz}(X)$ and per $W<$ per $X$. For $p \geq 6$, the matrix $X_{p}$ is the matrix obtained from $T_{p}$ by replacing the ones in positions $(2,3)$ and $(3,2)$ by zeros, and the zeros in positions $(5,3)$ and $(6,4)$ by ones. Then $\operatorname{nnz}\left(X_{p}\right)=\operatorname{nnz}\left(T_{p}\right)$, and on setting $f_{0}=1$ it follows that

$$
\begin{aligned}
\text { per } X_{p} & =2\left(f_{p-2}+f_{p-5}+f_{p-6}\right) \\
& =2\left(f_{p-2}+f_{p-4}\right) \\
& >2 f_{p-2}+f_{p-4}+f_{p-5} \\
& =2 f_{p-2}+f_{p-3}=f_{p}=\text { per } T_{p} .
\end{aligned}
$$

\begin{tabular}{|c|c|c|c|c|}
\hline$W$ & per $W$ & $X$ & per $X$ & Constraints \\
\hline$T_{1} \oplus T_{p}$ & $f_{p}$ & $T_{2} \oplus T_{p-1}$ & $2 f_{p-1}$ & $p \geq 3$ \\
\hline$T_{p}$ & $f_{p}$ & $X_{p}$ & $2 f_{p-2}+2 f_{p-4}$ & $p \geq 6$ \\
\hline$T_{5} \oplus T_{5}$ & 64 & $H_{3} \oplus H_{3} \oplus T_{4}$ & 80 & \\
\hline$T_{5} \oplus T_{4}$ & 40 & $H_{3} \oplus H_{3} \oplus T_{3}$ & 48 & \\
\hline$T_{5} \oplus T_{3}$ & 24 & $H_{3} \oplus H_{3} \oplus H_{2}$ & 32 & \\
\hline$T_{4} \oplus T_{4}$ & 25 & $H_{3} \oplus H_{3} \oplus H_{2}$ & 32 & \\
\hline$T_{4} \oplus T_{3}$ & 15 & $H_{3} \oplus H_{2} \oplus H_{2}$ & 16 & \\
\hline$T_{3} \oplus T_{3}$ & 9 & $H_{2} \oplus T_{4}$ & 10 & \\
\hline
\end{tabular}

Suppose that there is a direct sum of a subset of the fully indecomposable components of $A$ that is equal to a $W$ occuring in the chart. Then $W$ can be replaced in $A$ by $X$ to obtain a Hessenberg $(0,1)$-matrix $A^{\prime}$ of order $n$ with $\operatorname{nnz}\left(A^{\prime}\right) \leq \operatorname{nnz}(A)$ and per $A^{\prime}>$ per $A$, contradicting the fact that per $A=P(m, n)$. Hence no subset of the fully indecomposable components of $A$ has the form of a $W$ in the chart. Hence, $n_{i} \leq 5$ for all $i$, there is at most one $i$ with $n_{i} \in\{3,4,5\}$, and if there is an $i$ with $n_{i}=1$ then all remaining $n_{j}$ are at most 2 . Since $m \geq 2 n+1$, there is at least one $i$ with $n_{i} \geq 3$. Hence each $n_{i} \in\{2,3,4,5\}$, and at least one $n_{i}$ does not equal 2 since $m \geq 2 n+1$.

First suppose some $n_{i}=3$. Then $A$ is the direct sum of $T_{3}$ and $k \geq 1$ matrices $T_{2}$. It follows that $n=2 k+3, m=4 k+7=2 n+1, m \equiv 3 \bmod 4$ and per $A=2^{k} \times 3=$ $\frac{3}{2} \times 2^{(m-3) / 4}=u(m, n)$.

Next suppose some $n_{i}=4$. Then $A$ is the direct sum of $T_{4}$ and $k \geq 0$ matrices $T_{2}$. It follows that $n=2 k+4, m=4 k+10=2 n+2, m \equiv 2 \bmod 4$ and per $A=2^{k} \times 5=$ $\frac{5}{4} \times 2^{(m-2) / 4}=u(m, n)$.

Finally suppose some $n_{i}=5$. Then $A$ is the direct sum of $T_{5}$ and $k \geq 0$ matrices $T_{2}$. It follows that $n=2 k+5, m=4 k+13=2 n+3, m \equiv 1 \bmod 4$ and per $A=2^{k} \times 8=$ $2^{(m-1) / 4}=u(m, n)$.

Thus the result holds in this case.

Case 2: Statement (b) of Lemma 4.4 holds. 
Then there is an $A \in \mathcal{S}(m, n)$ none of whose fully indecomposable components belong to $\left\{T_{2}, T_{3}, T_{4}\right\}$. By Lemma 4.6,

$$
\text { per } A=\prod_{i=1}^{b} \text { per } A_{i} \leq \prod_{i=1}^{b} 2^{\mathrm{nnz}\left(A_{i}\right)-2 n_{i}}=2^{\mathrm{nnz}(A)-2 n}=2^{m-2 n} .
$$

Let $v$ be the unique integer $\in\{0,1,2,3\}$ such that $m \equiv v \bmod 4$. Since $m \leq 8 n / 3$, it follows that $3 m \leq 8 n-v$. If $v=0$, then $m-2 n \leq m-(3 / 4) m=m / 4$, and hence $2^{m-2 n} \leq 2^{m / 4}=u(m, n)$. If $v=1$, then $m-2 n \leq m-(3 m+1) / 4=(m-1) / 4$, and hence $2^{m-2 n} \leq 2^{(m-1) / 4}=u(m, n)$. If $v=2$, then $m-2 n \leq m-(3 m+2) / 4=(m-2) / 4$, and hence $2^{m-2 n} \leq 2^{(m-2) / 4}<(5 / 4) 2^{(m-2) / 4}=u(m, n)$. If $v=3$, then $m-2 n \leq$ $m-(3 m+3) / 4=(m-3) / 4$, and hence $2^{m-2 n} \leq 2^{(m-3) / 4}<(3 / 2) 2^{(m-3) / 4}=u(m, n)$.

Therefore the result holds for each $m$.

Case 3: Statement (c) of Lemma 4.4 holds.

Then there is $A \in \mathcal{S}(m, n)$ and an $i$ such that $A_{i}=H_{3}$. Let $A^{\prime}$ be the matrix obtained from $A$ by deleting the rows and columns that intersect $A_{i}$. Then $\operatorname{nnz}\left(A^{\prime}\right)=m-8, A^{\prime}$ is $(n-3)$-by- $(n-3)$ and per $A=4 \times$ per $A^{\prime}$. Since $m \leq 8 n / 3, \operatorname{nnz}\left(A^{\prime}\right)=m-8 \leq 8(n-3) / 3$.

First suppose that $m-8 \geq 2(n-3)+1$. Then by induction and the fact that $m \equiv m-8 \bmod 4$, per $A^{\prime} \leq u(m-8, n-3)=u(m, n) / 4$. As per $A=4 \times \operatorname{per} A^{\prime}$, the desired upper bounds now follow.

Now suppose that $m-8 \leq 2(n-3)$. As $m \geq 2 n+1$, it follows that $m=2 n+1$ or $m=2 n+2$. First consider $m=2 n+1$. Then $n n z\left(A^{\prime}\right)=2(n-3)-1<2(n-3)$. Hence, by Theorem 3.1, per $A^{\prime} \leq 2^{\lfloor(n-4) / 2\rfloor}=2^{\lfloor(m-9) / 4\rfloor}$. Thus, since $m$ is odd,

$$
\begin{aligned}
\text { per } A \leq 2^{\lfloor(m-1) / 4\rfloor} & = \begin{cases}2^{(m-1) / 4}, & \text { if } m \equiv 1 \bmod 4 \\
2^{(m-3) / 4}, & \text { if } m \equiv 3 \bmod 4,\end{cases} \\
& \leq u(m, n) .
\end{aligned}
$$

On the other hand, if $m=2 n+2$, then $\operatorname{nnz}\left(A^{\prime}\right)=2(n-3)$. Hence, by Theorem 3.1, per $A^{\prime} \leq 2^{\lfloor(n-3) / 2\rfloor}=2^{\lfloor(m-8) / 4\rfloor}$. Thus, since $m$ is even,

$$
\begin{aligned}
\text { per } A \leq 2^{\lfloor m / 4\rfloor} & =\left\{\begin{array}{cc}
2^{m / 4} & m \equiv 0 \bmod 4 \\
2^{(m-2) / 4} & m \equiv 2 \bmod 4,
\end{array}\right. \\
& \leq u(m, n) .
\end{aligned}
$$

Case 4: Statement (d) of Lemma 4.4 holds.

Then there is an $A \in \mathcal{S}(m, n)$ and an $i$ with $A_{i}=T_{3}$. If $A_{j}=T_{n_{j}}$ for all $j$, then Case 1 applies, and if some $A_{j}=H_{3}$, then Case 3 applies and per $A \leq u(m, n)$. Otherwise, there is a $j$ such that $A_{j}$ has a line with at least four entries equal to 1 . By Laplace expansion of per $A_{j}$ along such a line, per $A_{j}$ is a sum of at least 4 permanents of matrices of the form $A_{j}(r, s)$. Thus, for some $(r, s)$ such that the $(r, s)$-entry of $A_{j}$ is 1 , per $A_{j}(r, s) \leq$ per $A_{j} / 4$. Let $A^{\prime}$ be the matrix obtained from $A$ by changing the $(3,1)$-entry of $A_{i}$ to 
1 and the $(r, s)$-entry of $A_{j}$ to 0 . Note that per $A_{i}=$ per $T_{3}=3=(3 / 4)$ per $H_{3}$, and per $A_{j}-\operatorname{per} A_{j}(r, s) \geq 3$ per $A_{j} / 4$. Thus $\operatorname{nnz}\left(A^{\prime}\right)=\operatorname{nnz}(A)$, and

$$
\begin{aligned}
\text { per } A^{\prime} & =\operatorname{per} H_{3} \times\left[\operatorname{per} A_{j}-\operatorname{per} A_{j}(r, s)\right] \times \prod_{t \neq i, j} \operatorname{per} A_{t} \\
& \geq 4 \operatorname{per} T_{3} / 3 \times 3 \operatorname{per} A_{j} / 4 \times \prod_{t \neq i, j} \operatorname{per} A_{t} \\
& =\operatorname{per} A_{i} \times \operatorname{per} A_{j} \times \prod_{t \neq i, j} \operatorname{per} A_{t} \\
& =\operatorname{per} A .
\end{aligned}
$$

It follows that per $A^{\prime}=P(m, n)$, and hence $A^{\prime} \in \mathcal{S}(m, n)$. Since $A^{\prime}$ has $H_{3}$ as a fully indecomposable component, Case 3 applies.

Thus, in each case there exists a matrix in $\mathcal{S}(m, n)$ with permanent having $u(m, n)$ as an upper bound.

Combining Proposition 4.1 and Theorem 4.7 gives the main result of this section, with $u(m, n)$ as defined at the beginning of this section.

Corollary 4.8 If $m$ and $n$ are positive integers with $2 n+1 \leq m \leq 8 n / 3$, then $P(m, n)=$ $u(m, n)$.

\section{Dense Hessenberg matrices}

In this section we determine the exact values of $P(m, n)$ for all values of $m$ such that $m=\operatorname{nnz}\left(H_{n}\right)-z$ with $0 \leq z \leq \frac{k^{2}+3 k-2}{2}$ and $k=\lfloor n / 2\rfloor$. We begin with a result that gives the permanent of certain order $n-1$ submatrices of $H_{n}$.

Lemma 5.1 For $j \leq i$,

$$
\text { per } H_{n}(i, j)=\left\{\begin{array}{cl}
1 & \text { if } i=n \text { and } j=1 \\
2^{j-2} & \text { if } i=n \text { and } j \geq 2 \\
2^{n-i-1} & \text { if } n-1 \geq i \geq 1 \text { and } j=1 \\
2^{n-i+j-3} & \text { if } n-1 \geq i \geq 1 \text { and } j \geq 2
\end{array}\right.
$$

In addition, for $n-1 \geq i \geq 1$, per $H_{n}(i, i+1)=2^{n-2}$.

Proof. For $j \leq i$, the formula for per $H_{n}(i, j)$ follows from Lemma 2.2(c). Since $H_{n}(i, i+$ 1) $=H_{n-1}$, per $H_{n}(i, i+1)=2^{n-2}$.

Define $M_{n}=\left[m_{n}(i, j)\right]$ to be the $n$-by- $n$ matrix with $(i, j)$-entry equal to per $H_{n}(i, j)$ if $j \leq i+1$ and 0 otherwise. Note that $m_{n}(i, j)$ is equal to the number of transversals of 
$H_{n}$ that contain the $(i, j)$-entry. For example,

$$
M_{6}=\left[\begin{array}{cccccc}
16 & 16 & 0 & 0 & 0 & 0 \\
8 & 8 & 16 & 0 & 0 & 0 \\
4 & 4 & 8 & 16 & 0 & 0 \\
2 & 2 & 4 & 8 & 16 & 0 \\
1 & 1 & 2 & 4 & 8 & 16 \\
1 & 1 & 2 & 4 & 8 & 16
\end{array}\right] .
$$

Lemma 5.2 Let $A=\left[a_{i j}\right]$ be an n-by-n Hessenberg $(0,1)$-matrix. Then per $A \geq 2^{n-1}-$ $\sum_{\left\{(i, j): j \leq i+1, a_{i j}=0\right\}} m_{n}(i, j)$.

Proof. If $a_{i j}=0$, then the $m_{n}(i, j)$ transversals of $H_{n}$ that contain the $(i, j)$-entry are not transversals of $A$. Since some transversals may be counted more than once, there are at most

$$
\sum_{\left\{(i, j): j \leq i+1, a_{i j}=0\right\}} m_{n}(i, j)
$$

transversals of $H_{n}$ that are not transversals of $A$. The result now follows.

It is well known that every permutation can be expressed as the composition of disjoint cycles. In the following theorem, we identify a transversal $\tau$ with its permutation. Thus, by the cycles of $\tau$ we mean the cycles of the corresponding permutation.

Given a nonnegative integer $z$ with $z \leq \operatorname{nnz}\left(H_{n}\right)$, define $\sigma_{n z}$ to be the sum of the $z$ smallest nonzero entries of $M_{n}$. For example, $\sigma_{60}=0, \sigma_{61}=1, \sigma_{62}=2, \sigma_{63}=3$, $\sigma_{64}=4, \sigma_{65}=6$ and $\sigma_{66}=8$. The following theorem determines $P(m, n)$ for $n$ even and $m \geq \frac{3 n^{2}+6 n}{8}$, i.e., for a dense Hessenberg matrix of even order.

Theorem 5.3 Let $n=2 k \geq 4$ be an even positive integer, and let $z$ be an integer with $0 \leq z \leq \frac{k^{2}+3 k-2}{2}$. Then $P\left(n n z\left(H_{n}\right)-z, n\right)=2^{n-1}-\sigma_{n z}$.

Proof. When $n=2 k=4$, it is easily verified that:

$P(9,4)=4$, with equality for $A=\left(H_{2} \oplus H_{2}\right)+E_{32}$;

$P(10,4)=5$, with equality for $A=T_{4}$;

$P(11,4)=6$, with equality for $A=H_{4}-\left(E_{41}+E_{42}\right)$;

$P(12,4)=7$, with equality for $A=H_{4}-E_{41}$;

$P(13,4)=8$, with equality for $A=H_{4}$.

Note that $P(9,4)$ is not achieved by any fully indecomposable matrix. In the remainder of the proof, assume that $n \geq 6$.

Let $\mathcal{I}$ be the set of all pairs of integers $(i, j)$ with $n \geq i>j \geq 1$ and $(i, j) \neq(k+1, k)$. For each $(i, j) \in \mathcal{I}$, let $\mathcal{S}_{i j}$ denote a fixed set of transversals of $H_{n}$ such that the sets $\mathcal{S}_{i j}$ are mutually disjoint and each element of $\mathcal{S}_{i j}$ contains the $(i, j)$-entry. With regard to any such sets $\mathcal{S}_{i j}$, an upper bound is now obtained (see (9) below) for the permanent of an $n$-by- $n$ Hessenberg $(0,1)$-matrix $A=\left[a_{i j}\right]$ for which $a_{i, i+1}=1(i=1,2, \ldots, n-1), a_{i i}=1$ 
$(i=1,2, \ldots, n)$ and $a_{k+1, k}=1$ (and all other $a_{i j}$ with $i>j$ are 0 or 1 ). Note that each

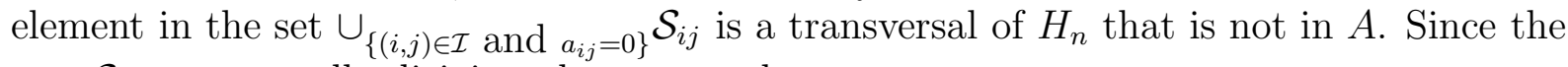
sets $\mathcal{S}_{i j}$ are mutually disjoint, there are at least

$$
\sum_{\left\{(i, j) \in \mathcal{I} \text { and } a_{i j}=0\right\}}\left|\mathcal{S}_{i j}\right|
$$

transversals of $H_{n}$ that are not transversals of $A$. Hence,

$$
\text { per } A \leq 2^{n-1}-\sum_{\left\{(i, j) \in \mathcal{I} \text { and } a_{i j}=0\right\}}\left|\mathcal{S}_{i j}\right| .
$$

We construct a family of such sets $\mathcal{S}_{i j}$ as follows. There are 4 types of pairs $(i, j)$ :

Type A: $i \geq k+1, k \geq j$ and $(i, j) \neq(k+1, k)$;

Type B: $i>j \geq k+1$;

Type C: $k-1 \geq i>j$;

Type D: $k=i>j$.

For $(i, j)$ of type $\mathrm{A}$, let $\mathcal{S}_{i j}$ consist of all transversals of the matrix

$$
\left[\begin{array}{ccc}
H_{j-1} & O & O \\
O & C_{i-j+1} & O \\
O & O & H_{n-i}
\end{array}\right]
$$

where $C_{i-j+1}$ is the cycle matrix defined in Section 4 and $H_{j-1}\left(H_{n-i}\right)$ is vacuous if $j=1$ $(i=n)$. We make the following observations if $(i, j)$ is of Type A:

(A1) If $\tau \in \mathcal{S}_{i j}$, then the cycle of $\tau$ that contains $k$ also contains $k+1$, but is not the 2-cycle $(k, k+1)$

(A2) Each transversal of $H_{n}$ (and thus of every $\mathcal{S}_{i j}$ ) contains at most one cycle that has an entry in $\langle 1, k\rangle$ and an entry in $\langle k+1, n\rangle$;

(A3) By (A1) and (A2), the sets $\mathcal{S}_{i j}$ of Type A are mutually disjoint;

(A4) If $(i, j)$ is of type $\mathrm{A}$, then

$$
\begin{aligned}
\left|\mathcal{S}_{i j}\right|= & \text { per } H_{j-1} \text { per } \\
= & H_{n-i} \\
= & \begin{array}{cl}
1 & \text { if } i=n \text { and } j=1, \\
2^{j-2} & \text { if } i=n \text { and } k \geq j \geq 2, \\
2^{n-i-1} & \text { if } n-1 \geq i \geq k+1 \text { and } j=1, \\
2^{n-i+j-3} & \text { if } n-1 \geq i \geq k+1 \text { and } k \geq j \geq 2,(i, j) \neq(k+1, k) .
\end{array}
\end{aligned}
$$


For $(i, j)$ of type $\mathrm{B}$, let $\mathcal{S}_{i j}$ be the set of all transversals of the matrix

$$
\left[\begin{array}{cccc}
H_{k-1} & O & O & O \\
0 & I_{j-k} & O & O \\
O & O & C_{i-j+1} & O \\
O & O & O & I_{n-i}
\end{array}\right]
$$

where $H_{k-1}\left(I_{n-i}\right)$ is vacuous if $k=1(i=n)$. We make the following observations if $(i, j)$ is of type $\mathrm{B}$ :

(B1) If $\tau \in \mathcal{S}_{i j}$, then $\tau$ contains the 1-cycle $(k, k)$;

(B2) $(i, j, \ldots, i-1)$ is the unique cycle of $\tau$ of length at least 2 with all of its elements in $\langle k+1, n\rangle$;

(B3) By (B2), the sets $\mathcal{S}_{i j}$ of type B are disjoint, and by (B1) and (A1), any set $\mathcal{S}_{i j}$ of type A and any set $\mathcal{S}_{i^{\prime} j^{\prime}}$ of type B are disjoint;

(B4) $\left|\mathcal{S}_{i j}\right|=2^{k-2}$.

For $(i, j)$ of type $\mathrm{C}$, let $\mathcal{S}_{i j}$ be the set of all transversals of the matrix

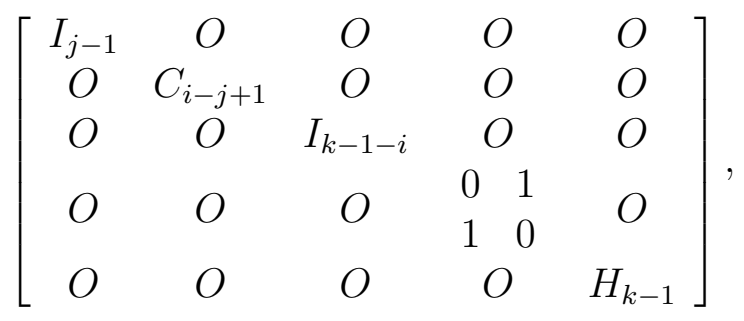

where $I_{j-1}\left(I_{k-1-i} ; H_{k-1}\right)$ is vacuous if $j=1(i=k-1 ; k=1)$. We make the following observations if $(i, j)$ is of Type $\mathrm{C}$ :

(C1) If $\tau \in \mathcal{S}_{i j}$, then the cycle of $\tau$ that contains $k$ is the 2-cycle $(k, k+1)$;

(C2) If $\tau \in \mathcal{S}_{i j}$, then the unique cycle of length at least 2 with all of its elements in $\langle 1, k-1\rangle$ is $(i, j, \ldots, i-1)$;

(C3) By (C2), the sets $\mathcal{S}_{i j}$ of types $\mathrm{C}$ are disjoint. By (A1), (B1) and (C1), any set $\mathcal{S}_{i j}$ of type $\mathrm{C}$ and any set $\mathcal{S}_{i^{\prime} j^{\prime}}$ of type A or B are disjoint;

(C4) $\left|\mathcal{S}_{i j}\right|=2^{k-2}$.

Finally, for $(i, j)$ of type $\mathrm{D}$, let $\mathcal{S}_{i j}$ be the set of all transversals of the matrix

$$
\left[\begin{array}{ccc}
I_{j-1} & O & O \\
O & C_{k-j+1} & O \\
O & O & H_{k}
\end{array}\right]
$$

where $I_{j-1}$ is vacuous if $j=1$. We make the following observations if $(i, j)$ is of type D: 
(D1) If $\tau \in \mathcal{S}_{i j}$, then the cycle of $\tau$ that contains $k$ has length at least 2 and each of its entries is in $\langle 1, k\rangle$.

(D2) $(k, j, j+1, \ldots, k-1)$ is the unique cycle of $\tau$ of length at least 2 with all of its elements in $\langle 1, k\rangle$;

(D3) By (D2), the sets $\mathcal{S}_{i j}$ of type D are disjoint. By (A1), (B1), (C1) and (D1), any set $\mathcal{S}_{i j}$ of type D and any set $\mathcal{S}_{i^{\prime} j^{\prime}}$ of type A, B or C are disjoint;

(D4) $\left|\mathcal{S}_{i j}\right|=2^{k-1}$.

Let $S_{n}$ be the $n$-by- $n$ matrix with $(i, j)$-entry equal to $\left|\mathcal{S}_{i j}\right|$ when $\mathcal{S}_{i j}$ is defined, and 0 otherwise. For example, for $n=6$

$$
S_{6}=\left[\begin{array}{cccccc}
0 & 0 & 0 & 0 & 0 & 0 \\
2 & 0 & 0 & 0 & 0 & 0 \\
4 & 4 & 0 & 0 & 0 & 0 \\
2 & 2 & 0 & 0 & 0 & 0 \\
1 & 1 & 2 & 2 & 0 & 0 \\
1 & 1 & 2 & 2 & 2 & 0
\end{array}\right]
$$

Fix $z$ to be an integer with $0 \leq z \leq \frac{k^{2}+3 k-2}{2}$, and let $A$ be an $n$-by- $n$ Hessenberg $(0,1)$-matrix with $z$ entries on or below the superdiagonal equal to 0 such that per $A=$ $P\left(\operatorname{nnz}\left(H_{n}\right)-z, n\right)$. By Theorem 2.3, we may assume that each fully indecomposable component of $A$ is Hessenberg and staircased.

We claim that $A$ is fully indecomposable. Suppose to the contrary that $A$ is not fully indecomposable; then without loss of generality, $A$ is in the form (1) with $b \geq 2$ and per $A \leq 2^{n-b}$. Let $H^{\prime}$ be the matrix obtained from $H_{k} \oplus H_{k}$ by replacing the $(k+1, k)$ and $(k, k+1)$ entries by ones. Then per $A \leq 2^{n-2}=\operatorname{per}\left(H_{k} \oplus H_{k}\right)<\operatorname{per} H^{\prime} \leq P\left(k^{2}+3 k, n\right) \leq$ $P\left(\operatorname{nnz}\left(H_{n}\right)-z, n\right)$, where the last inequality follows as $k \geq 3$. This contradicts the assumption that per $A=P\left(\operatorname{nnz}\left(H_{n}\right)-z, n\right)$, so $A$ is fully indecomposable.

By Lemma 2.2(b), $A \geq T_{n}$ (entrywise). Thus if $i>j$ and $a_{i j}=0$, then $(i, j) \in \mathcal{I}$. Hence by (9),

$$
\text { per } A \leq 2^{n-1}-\sum_{\left\{(i, j) \in \mathcal{I} \text { and } a_{i j}=0\right\}}\left|\mathcal{S}_{i j}\right| \leq 2^{n-1}-s_{n z},
$$

where $s_{n z}$ is the sum of the $z$ smallest nonzero entries of $S_{n}$. Since $z \leq\left(k^{2}+3 k-2\right) / 2$, by Lemma 5.1 and (A4) above, the $z$ smallest nonzero entries of $S_{n}$ and $M_{n}$ agree. Hence, $s_{n z}=\sigma_{n z}$ and $P\left(\operatorname{nnz}\left(H_{n}\right)-z, n\right) \leq 2^{n-1}-\sigma_{n z}$. Since, by Lemma 5.2, $P\left(\operatorname{nnz}\left(H_{n}\right)-z, n\right) \geq$ $2^{n-1}-\sigma_{n z}$, it follows that $P\left(\operatorname{nnz}\left(H_{n}\right)-z, n\right)=2^{n-1}-\sigma_{n z}$.

A corresponding result that determines $P(m, n)$ for $n$ odd and $m \geq \frac{3 n^{2}+8 n+5}{8}$ is now derived. Let $n=2 k+1 \geq 3$ be an odd positive integer, and $z$ an integer with $0 \leq z \leq$ $\frac{k^{2}+3 k-2}{2}$. Modifying (as described below) the proof of Theorem 5.3 gives the values of $P\left(\operatorname{nnz}\left(H_{n}\right)-z, n\right)$. 
Define the types A, B, C and D as in the proof of Theorem 5.3, and the sets $\mathcal{S}_{i j}$ as before when $(i, j)$ is of type A or B. For $(i, j)$ of type C, we now define $\mathcal{S}_{i j}$ to be the set of all transversals of

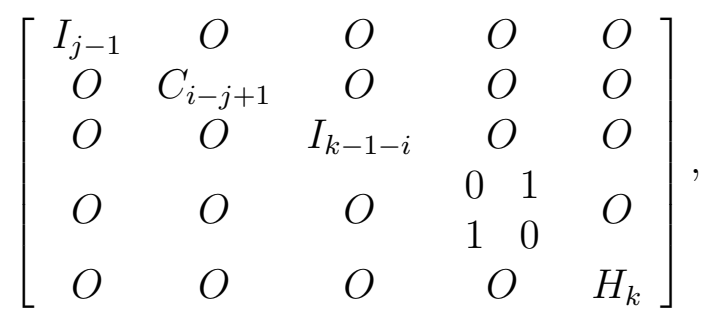

where $I_{j-1}\left(I_{k-1-i}\right)$ is vacuous if $j=1(i=k-1)$. For $(i, j)$ of type D, we now define $\mathcal{S}_{i j}$ to be the set of all transversals of

$$
\left[\begin{array}{ccc}
I_{j-1} & O & O \\
O & C_{k-j+1} & O \\
O & O & H_{k+1}
\end{array}\right]
$$

where $I_{j-1}$ is vacuous if $j=1$. Note that statements (A1)-(A4), (B1)-(B4), (C1)-(C3), (D1)-(D3) hold verbatim. Statement (C4) becomes $\left|\mathcal{S}_{i j}\right|=2^{k-1}$ and statement (D4) becomes $\left|\mathcal{S}_{i j}\right|=2^{k}$.

In modifying the penultimate paragraph of the proof of Theorem 5.3, let $H^{\prime}$ be obtained from $H_{k} \oplus H_{k+1}$ by replacing the $(k+1, k)$ and $(k, k+1)$ entries by ones. Then per $A \leq 2^{n-2}=\operatorname{per}\left(H_{k} \oplus H_{k+1}\right)<\operatorname{per} H^{\prime} \leq P\left(k^{2}+4 k+2, n\right) \leq P\left(\operatorname{nnz}\left(H_{n}\right)-z, n\right)$, where the last inequality follows if $k \geq 1$. These modifications to the proof of Theorem 5.3 give the following result.

Theorem 5.4 Let $n=2 k+1 \geq 3$ be an odd positive integer, and let $z$ be an integer with $0 \leq z \leq \frac{k^{2}+3 k-2}{2}$. Then $P\left(n n z\left(H_{n}\right)-z, n\right)=2^{n-1}-\sigma_{n z}$.

\section{Concluding Remarks}

For $n=3$ and $3 \leq m \leq 8$, our values of $P(m, n)$ are the same as the values given in $[\mathrm{BGM}]$ for the maximum permanent of an arbitrary 3-by-3 $(0,1)$-matrix with $m$ entries equal to 1 . However, for $n=4$ and $m=10$, this larger class can attain a maximum permanent of $6\left[\mathrm{BGM}\right.$, Table 1] given by $H_{1} \oplus J_{3}$, whereas $P(10,4)=5$.

Results from previous sections give $P(m, 2), P(m, 3)$ and $P(m, 4)$ for all possible values of $m$. For $n=5$, theorems from Sections 3 and 4 give $P(m, 5)$ for $5 \leq m \leq 13$, whereas values of $P(m, 5)$ for $m \geq 15$ are determined from Theorem 5.4. The value of $P(14,5)$ does not follow immediately from our theorems. However, we can use previous results on the staircase structure to determine the value of $P(14,5)$. If $A \in \mathcal{H}(14,5)$ is partly decomposable, then per $A \leq 2^{4-1}=8$. If $A \in \mathcal{H}(14,5)$ is fully indecomposable, then the diagonal, super- and sub-diagonal entries are all equal to 1 (accounting for 13 ones) and $A=T_{5}+E_{i j}$, with $(i, j) \in\{(3,1),(4,2),(5,3)\}$. Such a matrix $A$ has permanent equal to 9 or 10 , thus $P(14,5)=10$, with the maximum attained by $A=T_{5}+E_{31}$. In conclusion, we note in general that values of $P(m, n)$ for $8 n / 3<m<\left(n^{2}+3 n-2-\left(k^{2}+3 k-2\right)\right) / 2$, where $k=\lfloor n / 2\rfloor$, remain to be determined. 


\section{References}

[BGM] R. A. Brualdi, J.L. Goldwasser and T.S. Michael, Maximum permanents of matrices of zeros and ones. J. Combin. Theory, Ser. A 47, (1988), 207-245.

[BR] R. A. Brualdi and H. J. Ryser, Combinatorial Matrix Theory, Cambridge University Press, Cambridge, 1991.

[CW] G.-S. Cheon and I. M. Wanless, An update on Minc's survey of open problems involving permanents, Linear Alg. Appls., 403 (2005) 314-342.

[M] H. Minc, Permanents, in: Encyclopedia Math. Appl., vol. 6, Addison-Wesley, Reading, 1978.

[SHRC] Seok-Zun Song, Suk-Geun Hwang, Seog-Hoon Rim and Gi-Sang Cheon, Extremes of permanents of (0,1)-matrices, Linear Alg. Appls., 373 (2003), 197-210. 